In-band and out-of-band reflectance calibrations of the EUV multilayer mirrors of the Atmospheric Imaging Assembly instrument aboard the Solar Dynamics Observatory

R. Soufli, E. T. Al

August 20, 2012

Space Telescopes and Instrumentation 2012: Ultraviolet to Gamma Ray Amsterdam, Netherlands July 1, 2012 through July 6, 2012 
This document was prepared as an account of work sponsored by an agency of the United States government. Neither the United States government nor Lawrence Livermore National Security, LLC, nor any of their employees makes any warranty, expressed or implied, or assumes any legal liability or responsibility for the accuracy, completeness, or usefulness of any information, apparatus, product, or process disclosed, or represents that its use would not infringe privately owned rights. Reference herein to any specific commercial product, process, or service by trade name, trademark, manufacturer, or otherwise does not necessarily constitute or imply its endorsement, recommendation, or favoring by the United States government or Lawrence Livermore National Security, LLC. The views and opinions of authors expressed herein do not necessarily state or reflect those of the United States government or Lawrence Livermore National Security, LLC, and shall not be used for advertising or product endorsement purposes. 


\title{
In-band and out-of-band reflectance calibrations of the EUV multilayer mirrors of the Atmospheric Imaging Assembly instrument aboard the Solar Dynamics Observatory
}

\author{
Regina Soufli $^{1 *}$, Eberhard Spiller ${ }^{1}$, David L. Windt ${ }^{2}$, Jeff C. Robinson ${ }^{1}$, Eric M. Gullikson ${ }^{3}$, \\ Luis Rodriguez-de Marcos ${ }^{4}$, Mónica Fernández-Perea ${ }^{1}$, Sherry L. Baker ${ }^{1}$, \\ Andrew L. Aquila ${ }^{3 \dagger}$, Franklin J. Dollar ${ }^{3 \dagger}$, José Antonio Méndez ${ }^{4}$, Juan I. Larruquert ${ }^{4}$, \\ Leon Golub ${ }^{5}$, Paul Boerner ${ }^{6}$ \\ ${ }^{1}$ Lawrence Livermore National Laboratory, Livermore CA 94550, US \\ ${ }^{2}$ Reflective X-ray Optics LLC, New York, NY 10027, US \\ ${ }^{3}$ Lawrence Berkeley National Laboratory, Berkeley, CA 94720, US \\ ${ }^{4}$ GOLD-Instituto de Óptica-Consejo Superior de Investigaciones Científicas Serrano 144, 28006 \\ Madrid, Spain \\ ${ }^{5}$ Harvard-Smithsonian Center for Astrophysics, Cambridge, MA 02138, US \\ ${ }^{6}$ Solar and Astrophysics Laboratory, Lockheed Martin Advanced Technology \\ Center, Palo Alto, CA 94304, US
}

\begin{abstract}
Experimental multilayer reflectance data on flight mirrors and witnesses for three extreme ultraviolet (EUV) channels of the Atmospheric Imaging Assembly (AIA) instrument aboard NASA's Solar Dynamics Observatory are presented and compared to theoretical models. The relevance of these results to the performance of the AIA instrument is discussed.
\end{abstract}

Keywords: EUV multilayers, reflectance measurements, Solar Dynamics Observatory, Atmospheric Imaging Assembly.

\section{INTRODUCTION}

Launched on February 11, 2010, the Atmospheric Imaging Assembly (AIA) instrument ${ }^{1,2}$ aboard NASA's Solar Dynamics Observatory (SDO) ${ }^{3}$ is currently the most advanced solar mission in orbit, providing spectacular views of the solar corona (Fig. 1) by taking images that span 1.3 solar diameters at seven narrowband extreme ultraviolet (EUV) wavelength channels (centered at 93.9, 131.0, 171.1, 193.5, 211.3, 303.8 and 335.4 Å) and three ultraviolet (UV)/visible channels. The AIA instrument has a resolution of about 1 arcsecond, field of view of 41 arcminutes and a cadence of 1012 seconds and is observing the Sun 24 hours/day without interruption. The unique combination of spatial and temporal resolution, field of view and number of wavelengths of the AIA instrument are leading to unprecedented insights into the solar processes. New discoveries have already emerged based on AIA observations, such as the existence of "sympathetic flares" and the detection of quasi-periodic, high-speed solar waves. AIA is also providing clues towards solving the most intriguing enigma in modern solar physics: the origins and mechanisms of coronal heating. AIA aims in studying and elucidating the Sun's extremely complex and dynamic magnetic field, its plasma and related phenomena. The ultimate goal is to develop robust modeling and predictive capabilities of solar activity, including extreme events (such as flares and coronal mass ejections) which, when directed towards Earth, can disrupt satellite communications, electricity grids and may pose threats to aviation and astronaut safety. The AIA instrument was designed and built at the

\footnotetext{
*e-mail: regina.soufli@llnl.gov, phone: 925-422-6013.

$\dagger$ Andrew Aquila is currently with the European XFEL GmbH, 22607 Hamburg, Germany. Franklin J. Dollar is currently with the University of Michigan, Ann Arbor, MI 48109, US.
} 
Lockheed Martin Solar and Astrophysics Laboratory (LMSAL), part of Lockheed Martin Space Systems Company, Advanced Technology Center in Palo Alto, California. The AIA telescope assemblies were built at the Smithsonian Astrophysical Observatory under contract from Lockheed Martin.

The AIA is composed of four generalized Cassegrain telescopes, each including a primary-secondary pair of mirrors operating at near-normal angles of incidence. Each telescope images at two different wavelengths, accomplished by two different reflective EUV multilayer coatings, each deposited on half of the area of each mirror. As is shown in Fig. 2, the wavelength pairs assigned to each telescope are: 131.0 / 335.4 $\mathrm{A}$, (telescope 1), 211.3 / $193.5 \AA$ (telescope 2), UV / 171.1 $\AA$ (telescope 3), and 303.8 / $93.9 \AA$ (telescope 4). The EUV wavelengths were selected in order to observe the solar corona at specific temperatures corresponding to $\mathrm{Fe}$ and $\mathrm{He}\left(303.8 \AA\right.$ ) ion emission lines ${ }^{1}$, as shown in Table 1 and Fig. 1. The pairing of wavelengths in each telescope is such that either a $\mathrm{Zr}$ or an $\mathrm{Al}$ filter is sufficient for selection of one of the EUV wavelengths in the pair and suppression of the other, with the exception of the 193/211 A telescope where both wavelengths fall under the $\mathrm{Al}$ filter transmission range: in this case a hardware shutter is used as wavelength selector, as is illustrated in Fig 2. The development of multilayer coatings for each of the seven EUV channels for AIA has been discussed in detail in an earlier publication ${ }^{4}$. Ultimately, Mo/Y was selected for the $93.9 \AA$ A channel, Mo/Si was selected for the 131.0, 171.1, 193.5 and $211.3 \AA$ channels, and SiC/Si was selected for the 303.8 and $335.4 \AA$ channels. Initially, $\mathrm{Mg} / \mathrm{SiC}$ (with a unique combination of superior properties including near-zero stress, good spectral selectivity and higher reflectance compared to $\mathrm{SiC} / \mathrm{Si}$ ) had been selected for the 303.8 and $335.4 \AA$ channels ${ }^{4}$. However, the issue of $\mathrm{Mg}$ induced corrosion led to the rejection of $\mathrm{Mg} / \mathrm{SiC}$ and to its substitution with $\mathrm{SiC} / \mathrm{Si}$ on the AIA flight mirrors. Recently, corrosion-resistant, high-reflectance $\mathrm{Mg} / \mathrm{SiC}$ multilayers for the $250-800 \AA$ wavelength range have been developed and could enable use of $\mathrm{Mg} / \mathrm{SiC}$ as multilayer mirror coating in future EUV solar missions and in other EUV applications $s^{5,6,7,8}$.

Detailed quantitative analysis of data transmitted by the AIA instrument requires precise knowledge of the instrument response as a function of wavelength. Inaccuracies in the calibration can significantly complicate the interpretation of the observations. For example, if the calibration measurements underestimate the instrument's response to a particular emission line formed in the solar atmosphere at very low temperatures, then the emission from this "cool" line may be attributed to "hotter" material, leading to incorrect conclusions about the physical processes that govern the solar corona. The in-band EUV reflectance $\left(1^{\text {st }}\right.$ order Bragg peak) was mapped across the clear aperture of each AIA flight mirror as part of the calibration of the AIA instrument and the results are discussed in Section 3. For a given wavelength channel, out-of-band photons which are reflected by the multilayer mirrors and are not suppressed by the transmission filters aboard the AIA instrument, will reach the detectors and may introduce errors in the data analysis. Therefore, measurements of the instrument's sensitivity to out-of-band radiation for each channel are essential for improving the diagnostic capability of the AIA instrument. Measurements of the out-of-band multilayer reflectance in the wavelength range from 40 - $900 \AA$ A were recently obtained on AIA multilayer flight witness samples and are discussed in Section 4. Wavelengths longer than $900 \AA$ were not measured since the $\mathrm{Al}$ filters aboard the AIA instrument ${ }^{2}$ become strongly absorptive, thus preventing > $900 \AA$ A photons from reaching the AIA flight mirrors. Results from three AIA channels $(93.9,171.1$ and $335.4 \AA)$ are discussed in this manuscript, representative of the three multilayer material pairs $(\mathrm{Mo} / \mathrm{Y}$, $\mathrm{Mo} / \mathrm{Si}, \mathrm{SiC} / \mathrm{Si}$ ) aboard AIA. The complete set of measurements for all seven AIA wavelengths will be presented in an upcoming publication.

Table 1: Primary ion emission lines, their temperatures and related solar regions observed by AIA (from Ref. 1).

\begin{tabular}{|c|l|l|c|}
\hline Channel & Primary ion(s) & Region of atmosphere & Char. $\log (\mathbf{T})$ \\
\hline $4500 \AA$ & continuum & photosphere & 3.7 \\
\hline $1700 \AA$ & continuum & temperature minimum, photosphere & 3.7 \\
\hline $303.8 \AA$ & He II & chromosphere, transition region & 4.7 \\
\hline $1600 \AA$ & C IV+continuum & transition region, upper photosphere & 5.0 \\
\hline $171.1 \AA$ & Fe IX & quiet corona, upper transition region & 5.8 \\
\hline $193.5 \AA$ & Fe XII, XXIV & corona and hot flare plasma & $6.2,7.3$ \\
\hline $211.3 \AA$ & Fe XIV & active-region corona & 6.3 \\
\hline $335.4 \AA$ & Fe XVI & active-region corona & 6.4 \\
\hline $93.9 \AA$ & Fe XVIII & flaring corona & 6.8 \\
\hline $131 \AA$ & Fe VIII, XXI & transition region, flaring corona & $5.6,7.0$ \\
\hline
\end{tabular}



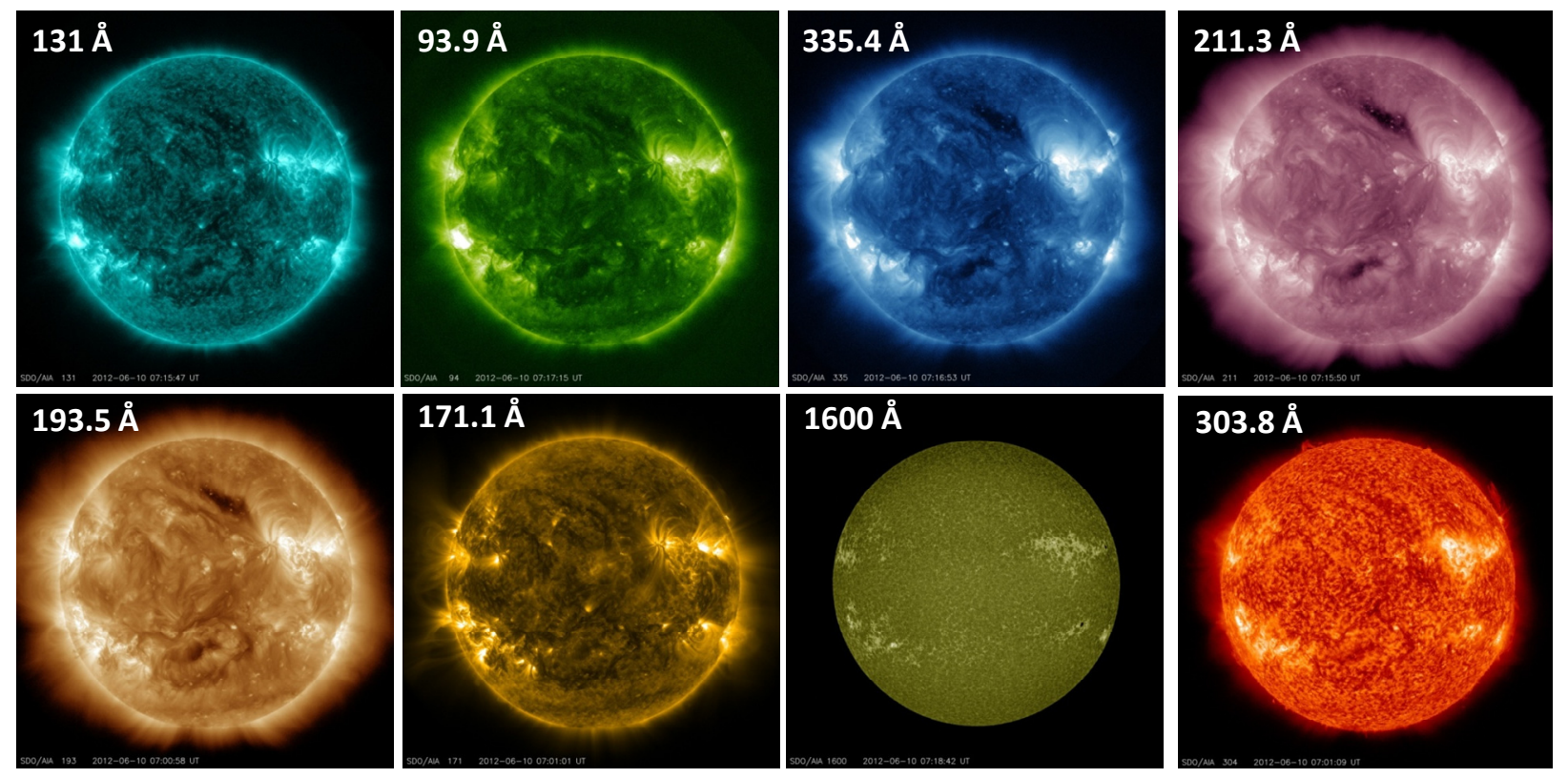

Fig. 1: (a) The Sun as observed by the AIA instrument at seven EUV channels and one UV channel (1600 ̊) on June 10, 2012. Wavelengths are displayed in approximate order of decreasing temperature, with $131 \AA$ being the highest-temperature and $303.8 \AA$ being the lowest-temperature emission line ${ }^{1}$ (see also Table 1). Images are courtesy NASA/LMSAL ${ }^{3}$.
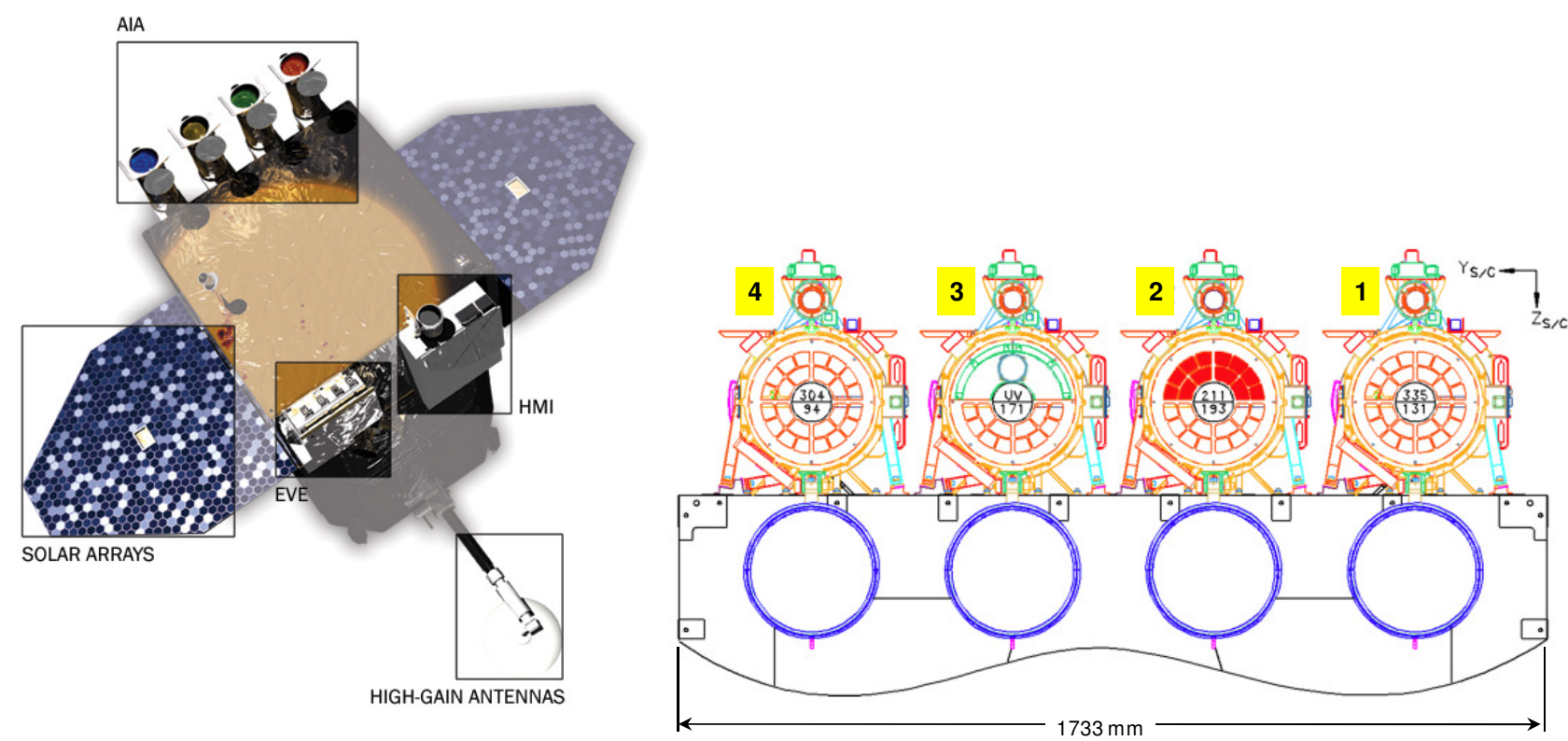

Fig. 2: Left: Image of SDO spacecraft with the AIA instrument shown, courtesy of NASA ${ }^{3}$.Two more instruments are part of SDO and are shown: the Extreme Ultraviolet Variability Experiment (EVE) and the Helioseismic and Magnetic Imager (HMI). Right: Detailed configuration of AIA telescopes. 


\section{EXPERIMENTAL FACILITIES}

The AIA flight multilayer mirrors and witnesses were coated in two deposition facilities, at Lawrence Livermore National Laboratory (LLNL) and at Reflective X-ray Optics (RXO). Regarding the three AIA multilayer channels that are discussed in this manuscript, the $171.1 \AA$ (Mo/Si) coatings were deposited at LLNL and the $93.9(\mathrm{Mo} / \mathrm{Y})$ and 335.4 $\AA$ ( $\mathrm{SiC} / \mathrm{Si}$ ) coatings were deposited at RXO. The LLNL deposition system is a planar DC-magnetron sputtering system with a "sputter down" geometry which has been described in detail earlier". An algorithm based on modulation of the rotational velocity of the deposition platter is used to control the coating thickness and to achieve the required coating thickness uniformity. Each sputtering source (cathode) has dimensions $12.7 \times 55.9 \mathrm{~cm}^{2}$. The Mo and Si sources were operated at a constant power of $800 \mathrm{~W}$ and $2000 \mathrm{~W}$, respectively. Base pressure was maintained at $2 \times 10^{-8}$ to $3 \times 10^{-8}$ Torr and the Ar process gas pressure was $1 \mathrm{mTorr}$. The multilayer coatings deposited at RXO were produced using a deposition system that has been described previously ${ }^{10}$. In this system 50 -cm-long planar cathodes are used. The spinning mirror substrate faces down and the magnetron cathodes sputter up. The individual layer thicknesses are determined by controlling the rotational velocity of the substrate as it passes over each cathode. For the coatings discussed here, the base pressure in the RXO chamber was less than $2 \times 10^{-7}$ Torr, the Ar process gas pressure was maintained at $1.6 \mathrm{mTorr}$, and the cathodes were operated in constant power mode: $\mathrm{SiC}$ and $\mathrm{Si}$ were deposited at $400 \mathrm{~W}$, Mo at $200 \mathrm{~W}$ and $\mathrm{Y}$ at $200 \mathrm{~W}$.

EUV reflectance measurements in the wavelength range 40-500 ̊ were performed at beamline 6.3.2. of the Advanced Light Source (ALS) synchrotron at Lawrence Berkeley National Laboratory. The general characteristics of the beamline have been described in detail earlier ${ }^{11,12}$. The sample chamber allows translation of the sample in three dimensions, tilt in two dimensions and azimuth rotation of the sample holder. The available detectors include a variety of photodiodes and a CCD camera (the latter for sample alignment), which can be rotated $360^{\circ}$ around the axis of the chamber. For the EUV reflectance measurements discussed in this manuscript, three gratings $(80,200$ and 600 lines $/ \mathrm{mm})$ were used in the monochromator. The monochromator exit slit was set to a width of $40 \mu \mathrm{m}$. Wavelength calibration was based on the $\mathrm{L}_{2,3}$ absorption edges of Al, Si and Ti transmission filters with a relative accuracy of $0.011 \% \mathrm{rms}$, and could be determined with $0.007 \%$ repeatability. During the measurements, $2^{\text {nd }}$ harmonic and stray light suppression was achieved with a series of transmission filters (Mg, Al, Si, B, C). For higher-order harmonic suppression, an "order suppressor" consisting of three mirrors at a variable grazing incidence angle (depending on wavelength range) and based on the principle of total external reflection was used in addition to the filters. The ALS storage ring current was used to normalize the signal against the storage ring current decay. For the in-band reflectance measurements on AIA flight mirrors, the signal was collected on a Si photodiode detector with $2.4^{\circ}$ angular acceptance. For the in-band and out-of-band reflectance measurements on AIA flight witness samples in the 40-500 $\AA$ wavelength range, the signal was collected on a GaAsP photodiode detector, with $1^{\circ}$ angular acceptance. All wavelength values were obtained within " $2 \sigma$ " error bars of $0.05 \%$. Reflectance was measured with $\pm 0.2 \%$ to $\pm 1 \%$ relative accuracy, determined by photodiode uniformity in each wavelength range. The vacuum pressure in the measurement chamber was in the range $2 \times 10^{-6}-10^{-7}$ Torr. Out-of-band reflectance measurements on AIA flight witness samples in the 487-895 ^ wavelength range were performed at the Grupo de Óptica de Laminas Delgadas (GOLD) facility at Instituto de Óptica. The reflectometer consists of a lamp and a grazing incidence toroidal-grating monochromator. The reflectometer covers the 125-2000 wavelength range. The monochromator has two Pt-coated diffraction gratings, 250 and 950 lines $/ \mathrm{mm}$. The entrance-exit arms are $146^{\circ}$ apart. A windowless discharge lamp is used to generate many spectral lines that cover the range longer than $\sim 400 \AA$. The lamp is fed with various pure gases such as $\mathrm{Ar}, \mathrm{He}$ or $\mathrm{CO}_{2}$, or gas mixtures consisting of various noble gases, along with $\mathrm{N}_{2}$ or $\mathrm{H}_{2}$. In most cases, radiation corresponds to a pure spectral line (with a low background) since the next spectral line is rejected with the spectral resolution of the monochromator. The beam divergence was $\sim 5 \mathrm{mrad}$ and angle accuracy is estimated as $\pm 0.1^{\circ}$. Samples up to $50.8 \times 50.8 \mathrm{~mm}^{2}$ size can be loaded in the sample holder, from which a rectangle of $50.8 \times 25 \mathrm{~mm}^{2}$ can be scanned. The detector is a channel electron multiplier with a CsI-coated photocathode. Reflectance was obtained by the successive measurement of the incident intensity and the intensity reflected by the sample. Reflectance accuracy is estimated as $\pm 1 \%$.

\section{IN-BAND REFLECTANCE CALIBRATIONS OF AIA FLIGHT MIRRORS}

The AIA flight mirror substrates are made of Zerodur ${ }^{\mathrm{TM}}$ material (see Fig. 3) and were figured and polished independently by two different optics vendors: Sagem Corporation (France) and L-3 Communications-Tinsley Laboratories (Richmond, California). For the flight mirrors that will be discussed in this manuscript, the primary flight substrates coated with the 93.9, 171.1, and 335.4 A multilayer coatings and the secondary flight substrates coated with 
the $171.1 \AA$ and $335.4 \AA$ multilayer coatings were fabricated by Sagem Corporation. The secondary flight substrate coated with the $93.9 \AA$ A multilayer coating was fabricated by L-3 Communications-Tinsley Laboratories. The high spatial frequency roughness (HSFR) of the mirror substrates, which can significantly affect the EUV reflective performance of the multilayer-coated mirrors, was measured by Atomic Force Microscopy at LLNL and the results are discussed in Ref. 13. Prior to multilayer coating, the flight substrates were cleaned at LLNL with a process customized for ultra-smooth mirror substrates. This process has been shown to remove polishing residue and contamination from the top surface which, if left untreated, could adversely affect and even cause delamination of the subsequently deposited multilayer reflective coating. The cleaning process does not degrade the surface finish ${ }^{13}$.

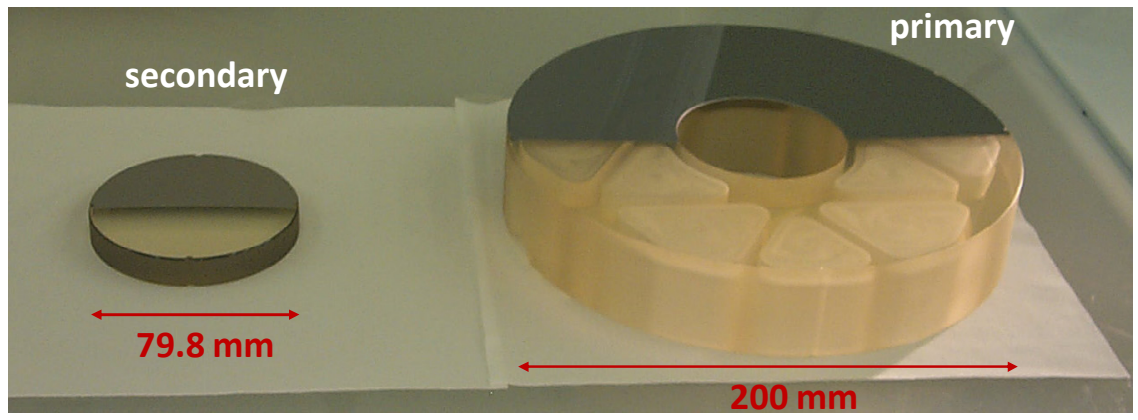

Fig. 3: A pair of AIA flight mirrors is shown, multilayer-coated at LLNL with Mo/Si at $171.1 \AA$. One half of each mirror is shown as un-coated Zerodur ${ }^{\mathrm{TM}}$ substrate and was coated later with a broadband UV reflective coating by a commercial vendor. The lightweighting structure is visible in the back of the primary mirror.

The prescription for the AIA primary and secondary flight mirror surfaces is given by:

$$
z(r)=\frac{1}{R} \cdot \frac{r^{2}}{\left[1+\sqrt{1-(1+K) \cdot(r / R)^{2}}\right]}
$$

where $\mathrm{z}$ is the surface height, $\mathrm{r}$ is the radial position defined from the optical axis of each mirror, $\mathrm{R}$ is the radius of curvature and $\mathrm{K}$ is the conic constant. Table 2 summarizes the values of $\mathrm{R}$ and $\mathrm{K}$, as well as the mirror and clear aperture radii and incidence angles for the AIA mirrors. A $66.5 \mathrm{~mm}$-diameter opening exists at the center of the primary substrates, for ray clearance. As is shown in Table 2, the angles of incidence for both mirrors are close to the normal direction $\left(90^{\circ}\right)$ and vary only slightly across the clear aperture. Therefore, uniform multilayer coatings were prescribed for both primary and secondary mirrors of the AIA instrument. The EUV reflectance was measured vs. wavelength on 78 locations across the surface of each multilayer-coated flight mirror. Fig. 4 shows a schematic diagram of the optic orientation and axes during the measurements. On the primary mirror, 6 radii $\mathrm{r}=45,60,75,90,92,94 \mathrm{~mm}$ were measured at 13 azimuth angles $\varphi= \pm 90^{\circ}, \pm 85^{\circ}, \pm 80^{\circ}, \pm 75^{\circ}, \pm 60^{\circ}, \pm 30^{\circ}, 0^{\circ}$. It should be noted that $\mathrm{r}=45,94 \mathrm{~mm}$ are at the edge of the clear aperture of the primary mirror. Furthermore, due to the specified "dead strip" width of $3.05 \mathrm{~mm}$ across the center of the primary mirror, $\varphi= \pm 90^{\circ}$ is outside the clear aperture for all radii. On the secondary mirror, 6 radii $\mathrm{r}=9,12,20,30,32,34 \mathrm{~mm}$ were measured at 13 azimuth angles $\varphi= \pm 90^{\circ}, \pm 85^{\circ}, \pm 80^{\circ}, \pm 75^{\circ}, \pm 60^{\circ}, \pm 30^{\circ}, 0^{\circ}$. It should be noted that $r=9,34 \mathrm{~mm}$ are at the edge of the clear aperture and, due to the specified "dead strip" width of 1 $\mathrm{mm}$ across the center of the secondary mirror, $\varphi= \pm 90^{\circ}$ is outside the clear aperture for all radii and $\varphi= \pm 85^{\circ}$ is outside the clear aperture for $\mathrm{r}=9 \mathrm{~mm}$. The measurement angle for all flight mirrors was set at angle $\theta_{\text {meas }}=87^{\circ}$ (from the grazing direction) and was later translated to the actual AIA angles of incidence, shown in Table 1. Geometrical constraints inside the reflectometer prevented measurements at the actual AIA angles of incidence.

The multilayer wavelength (or thickness) uniformity specification for each AIA flight mirror was as follows: peak wavelength should remain within $\pm(0.3 \times$ FWHM $)$ of the goal wavelength for a given AIA channel, in $80 \%$ of the clear aperture (defined in Table 2 for each mirror), where FWHM is the full-width-half-maximum of the multilayer EUV reflectance curve. In addition to the challenge of achieving the aforementioned multilayer thickness uniformity on a curved mirror surface, the D-shaped hardware "mask" that had to be placed on each mirror (to protect half of the mirror while the other half was being coated), combined with the hardware coating fixtures that hold the optic during 
deposition, posed an additional challenge: hardware parts located in the vicinity of the surface that is being coated, cause sputtered species directed towards the substrate to be scattered in different directions, resulting in multilayer thin film coatings with unwanted thickness variations and reduced peak reflectance in those areas. This phenomenon is known as "shadowing" and required special considerations in the fabrication of the hardware mask and coating fixtures, in order to minimize these detrimental effects. Multilayer reflectance has also a pronounced dependence on substrate HSFR at EUV wavelengths. Based on the range of HSFR values of the AIA flight substrates and on the brightness of each solar emission line to be imaged by AIA, a "goal" for the peak reflectance of the flight mirrors was set for each AIA channel. The goal for the AIA flight mirror peak reflectance was $>20 \%$ at $93.9 \AA,>35 \%$ at $171.1 \AA$ and $>10 \%$ at $335.4 \AA$, in > $80 \%$ of the clear aperture. A specification for the peak multilayer reflectance assuming nearly-ideal substrates was also set for each AIA channel, as will be discussed in Section 4.

Figs. 5, 6, and 7, show the measured EUV reflectance results for the 93.9, 171.1 and $335.4 \AA$ multilayer-coated flight mirror pairs of the AIA instrument. The flight mirrors comply with the AIA peak wavelength uniformity and peak reflectance specifications discussed in the previous paragraph. These measurements were combined using an areaweighted average to produce a single composite reflectivity function for each mirror that was used in the initial calibration of the AIA instrument ${ }^{2}$.

Table 2: The geometrical parameters of the AIA flight mirrors discussed in this manuscript are shown. The "dead strip" is an area (defined from the center line of each mirror), which is allowed to remain uncoated or partially coated. Any coating located within the "dead strip" does not have to comply with the AIA multilayer specifications. Angles of incidence are defined from the grazing direction. Optical design by W. A. Podgorski (Smithsonian Astrophysical Observatory).

\begin{tabular}{|c|c|c|}
\hline & Primary (concave) & Secondary (convex) \\
\hline $\mathbf{R}(\mathbf{m m})$ & $2755.01 \pm 1$ & $1208.57 \pm 0.4$ \\
\hline $\mathbf{K}$ & -1.0916 & -5.0766 \\
\hline Mirror outer radius (mm) & 100.0 & 39.9 \\
\hline $\begin{array}{c}\text { Clear aperture } \\
\text { outer radius (mm) }\end{array}$ & 94.0 & 34.0 \\
\hline $\begin{array}{c}\text { Clear aperture } \\
\text { inner radius (mm) }\end{array}$ & 45.1 & 9.0 \\
\hline "Dead strip” width (mm) & 3.05 & 1.0 \\
\hline $\begin{array}{c}\text { Range of incidence angles } \\
\text { within clear aperture }\end{array}$ & $89.1^{\circ}-88.0^{\circ}$ & $90.4^{\circ}-91.8^{\circ}$ \\
\hline
\end{tabular}

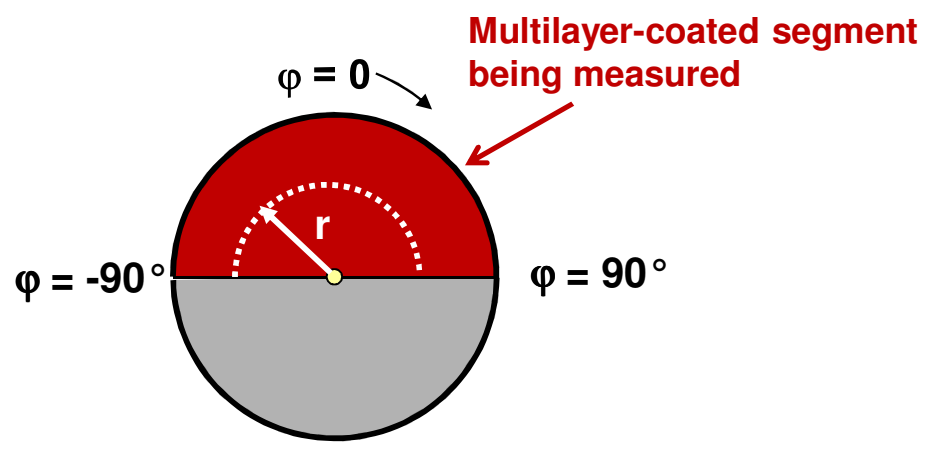

Fig. 4: Schematic drawing of AIA flight mirror during EUV reflectance measurements with $r$ (radial distance) and $\varphi$ (azimuth angle) axes shown. 

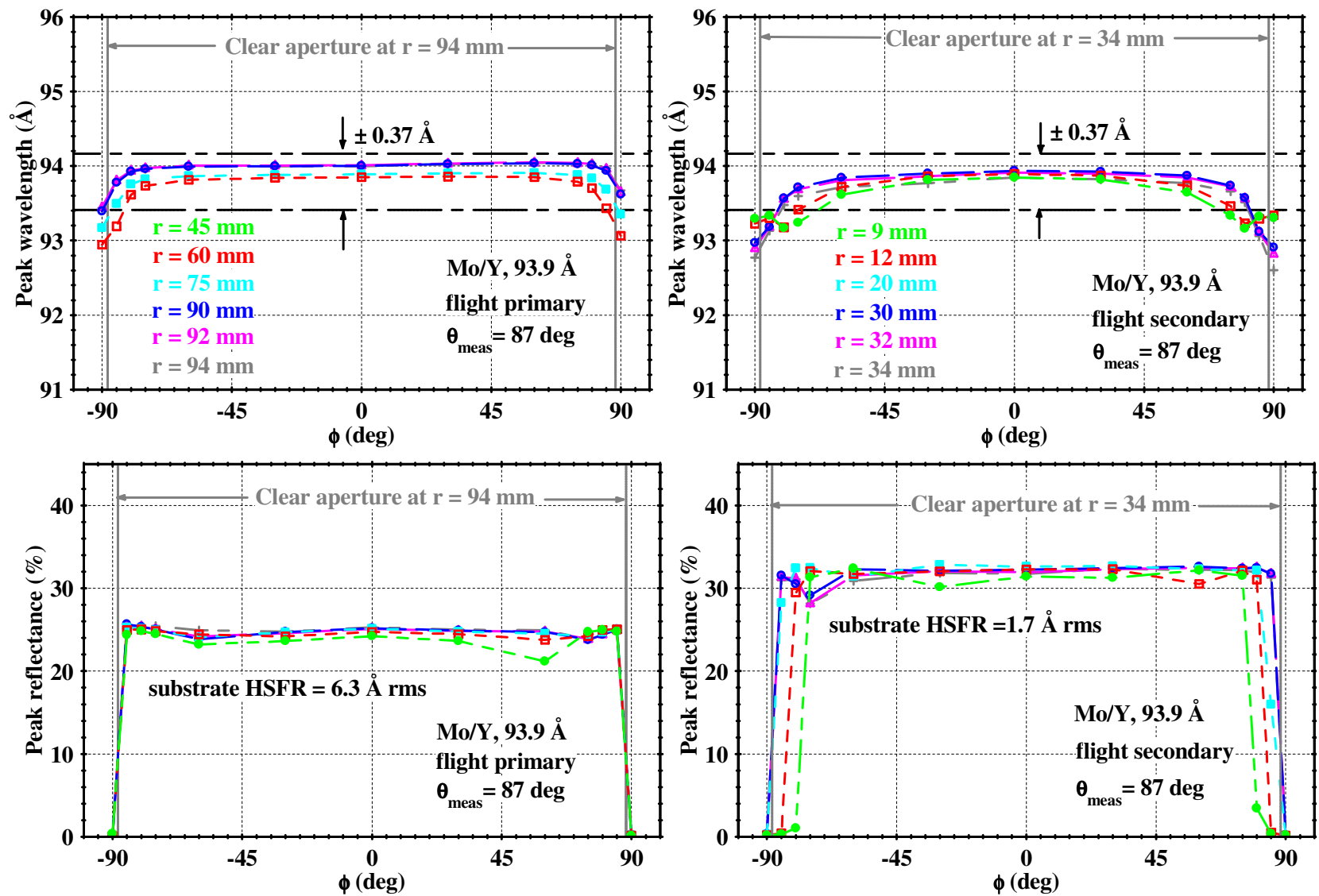

Fig. 5: Peak wavelength and peak reflectance (extracted from EUV reflectance vs. wavelength curves) of AIA flight primary (left) and secondary (right) multilayer mirrors at $93.9 \AA$, measured at 78 locations across the surface of each mirror at ALS beamline 6.3.2. The mirror geometry and axes during the measurements are shown in Fig. 4. The Mo/Y multilayer coating consists of $\mathrm{N}=120 \mathrm{Mo} / \mathrm{Y}$ bilayers with period $=47.8 \AA, \Gamma=0.35$ (ratio of Mo thickness in the bilayer) and a $16.7 \AA$-thick Mo capping layer. The mirror substrate HSFR was $6.3 \AA$ rms (primary) and $1.7 \AA \mathrm{rms}$ (secondary), measured by atomic force microscopy at LLNL. The multilayer-coated AIA flight mirrors are well within the peak wavelength tolerance of $93.9 \pm 0.37 \AA$ and peak reflectance goal of $>20 \%$, required in $>$ $80 \%$ of the clear aperture.

\section{IN-BAND AND OUT-OF-BAND REFLECTANCE CALIBRATIONS OF AIA FLIGHT WITNESS SAMPLES}

To allow for long-term monitoring of the lifetime properties of the AIA flight multilayer coatings, flight "witness" multilayer coatings were deposited during each flight deposition run on $\sim 525 \mu \mathrm{m}$ thick $\mathrm{Si}(100)$ wafer substrates with HSFR $<1 \AA$ rms. Some of the witnesses were stored in special containers with the AIA flight mirrors until the mirrors were installed in the AIA instrument aboard SDO. Other flight witness pieces, with dimensions around $20 \times 20 \mathrm{~mm}^{2}$, were stored in laboratory environment (air). The in-band and out-of-band reflectance of the AIA flight witnesses stored in air was measured in the 40-500 $\AA$ wavelength range at beamline 6.3.2. of the ALS, 3-4 years after deposition. The out-ofband reflectance of the same AIA flight witnesses was also measured in the 487-895 $\AA$ wavelength range at the GOLD facility, 5-6 years after deposition. ALS measurements were performed at $87^{\circ}$ incidence angle to match the measurement angle of the AIA flight mirrors, while GOLD measurements were performed at $85^{\circ}$. The difference in measured multilayer reflectance between these two near-normal incidence angles is expected to be negligible. The reflectance measurement results for the 93.9, 171.1 and 335.4 A AIA channels are plotted in Figs. 8, 9, and 10. The results demonstrate excellent agreement in overlapping wavelength ranges between the ALS and GOLD facilities. 

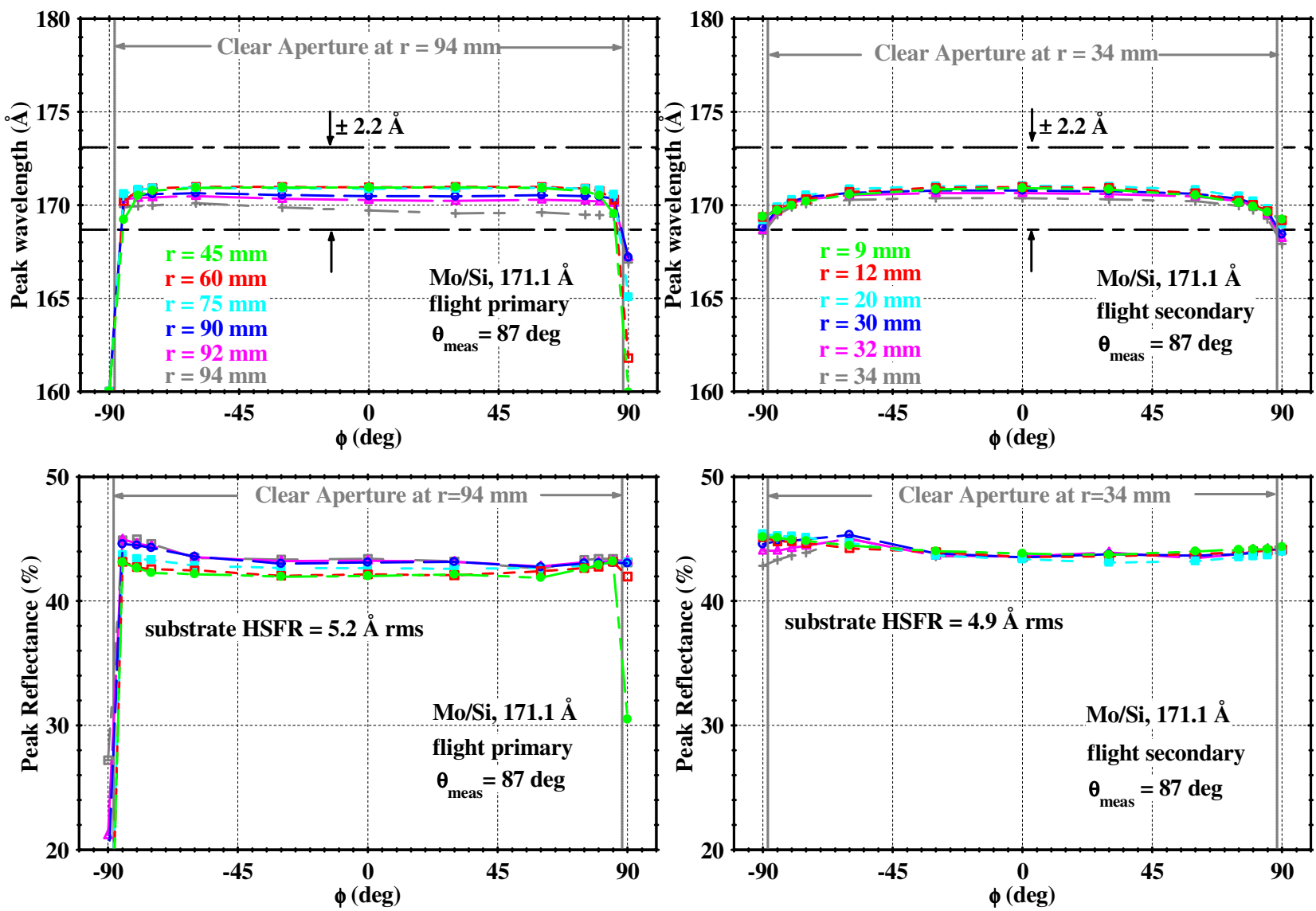

Fig. 6: Peak wavelength and peak reflectance (extracted from EUV reflectance vs. wavelength curves) of AIA flight primary (left) and secondary (right) multilayer mirrors at $171.1 \AA$, measured at 78 locations across the surface of each mirror at ALS beamline 6.3.2. The mirror geometry and axes during the measurements are shown in Fig. 4. The Mo/Si multilayer coating consists of $\mathrm{N}=40 \mathrm{Mo} / \mathrm{Si}$ bilayers with period $=88.4 \AA, \Gamma=0.17$ (ratio of Mo thickness in the bilayer) and a $35 \AA$-thick Si capping layer. The mirror substrate HSFR was 5.2 A rms (primary) and $4.9 \AA \mathrm{rms}$ (secondary), measured by atomic force microscopy at LLNL. The multilayer-coated AIA flight mirrors are well within the peak wavelength tolerance of $171.1 \pm 2.2 \AA$ and peak reflectance goal of $>30 \%$, required in $>$ $80 \%$ of the clear aperture.

It is also demonstrated that the out-of-band reflectance of all three AIA multilayers is significant (10\% or higher) at the longer wavelengths approaching $900 \AA$. A model for the measured reflectance was also constructed for each of the $\mathrm{Mo} / \mathrm{Y}, \mathrm{Mo} / \mathrm{Si}$ and $\mathrm{SiC} / \mathrm{Si}$ multilayer coatings, using the IMD program ${ }^{14}$ and a compilation of experimental optical constants (refractive index) values. More specifically, for the interior Mo layers in the $\mathrm{Mo} / \mathrm{Y}$ and $\mathrm{Mo} / \mathrm{Si}$ multilayer models, the optical constants compilation from the 1993 Atomic Tables ${ }^{15}$ was combined with more recent experimental Mo data ${ }^{16,17,18}$. For the top (capping) Mo layer in the Mo/Y multilayer model, experimental optical constants from Ref. 19, which include the presence of Mo oxides on the top Mo surface (which is exposed to air) were used. For the Y layers in the $\mathrm{Mo} / \mathrm{Y}$ multilayer model, experimental data from Refs. 20 and 21 were used. For the Si substrate model in all three samples, for the Si layers (including the capping layer) in the Mo/Si multilayer model, and for the Si layers in the $\mathrm{SiC} / \mathrm{Si}$ multilayer model, the optical constants compilation from the 1993 Atomic Tables ${ }^{15}$ was combined with other experimental $\mathrm{Si}$ data ${ }^{22,23,24,25}$. A thin $\mathrm{SiO}_{2}$ layer was included on top of the $\mathrm{Si}$ capping layer in the $\mathrm{Mo} / \mathrm{Si}$ multilayer model and on top of the Si substrate model, with optical constants from Refs. 26, 27 and 28. For the inner SiC layers in the $\mathrm{SiC}$ multilayer model, experimental optical constants from Ref. 29 were used. For the top (capping) SiC layer in the $\mathrm{SiC} / \mathrm{Si}$ multilayer model, experimental optical constants from Refs. 29 and 30 (the latter including the presence of Si oxides on the top $\mathrm{SiC}$ surface) were used. For the $\mathrm{Cr}$ underlayer in the $\mathrm{SiC} / \mathrm{Si}$ multilayer model, the optical constants compilation from the 1993 Atomic Tables ${ }^{15}$ was combined with data from Ref. 18. 

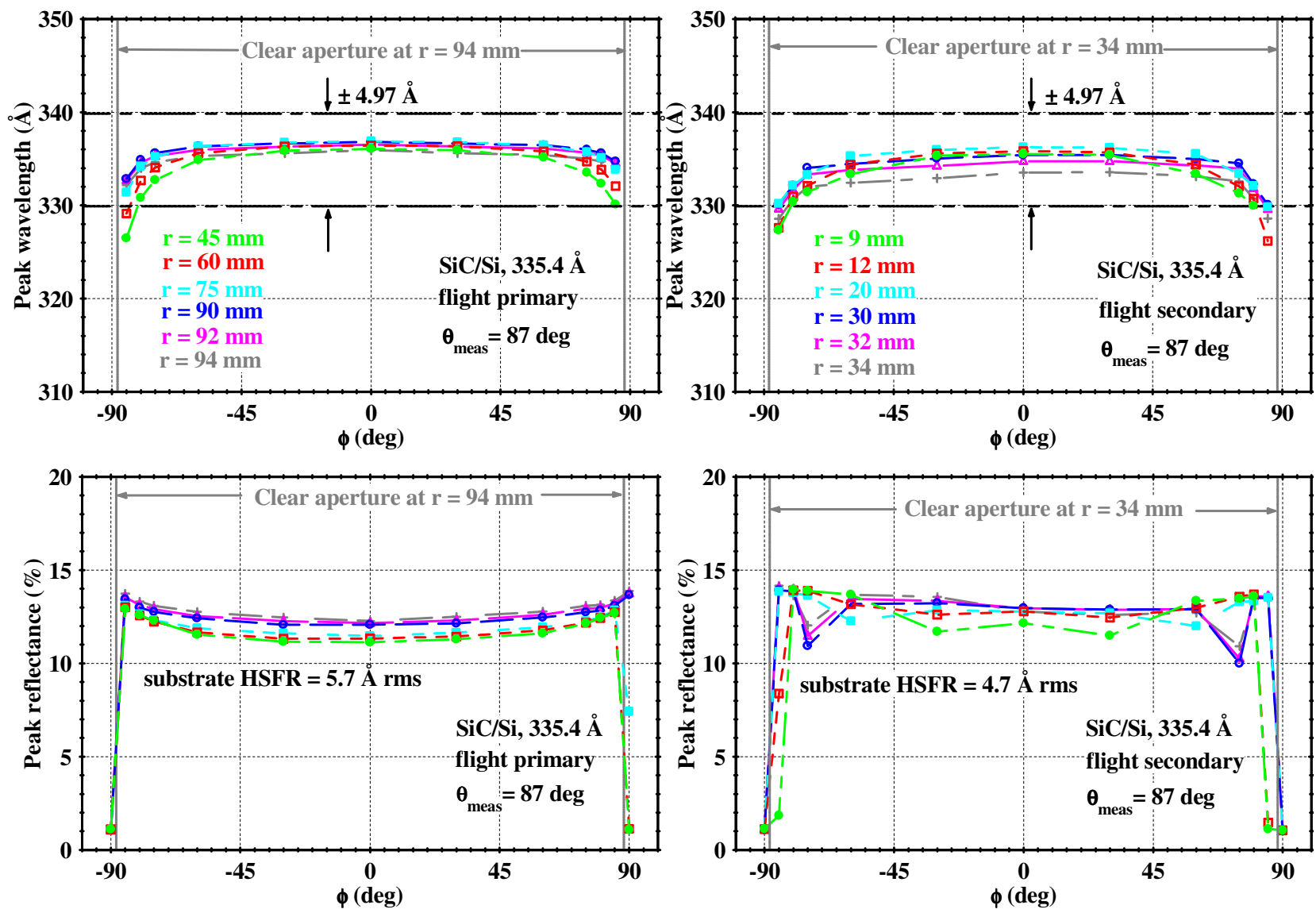

Fig. 7: Peak wavelength and peak reflectance (extracted from EUV reflectance vs. wavelength curves) of AIA flight primary (left) and secondary (right) multilayer mirrors at 335.4 A, measured at 78 locations across the surface of each mirror at ALS beamline 6.3.2. The mirror geometry and axes during the measurements are shown in Fig. 4. The $\mathrm{SiC} / \mathrm{Si}$ multilayer coating consists of $\mathrm{N}=30 \mathrm{SiC} / \mathrm{Si}$ bilayers with period $=187.8 \AA, \Gamma=0.25$ (ratio of $\mathrm{SiC}$ thickness in the bilayer) and a $47 \AA$-thick SiC capping layer. A $200 \AA$-thick Cr adhesion layer was also deposited between the multilayer coating and the mirror substrate. The mirror substrate HSFR was $5.7 \AA$ rms (primary) and $4.7 \AA \mathrm{rms}$ (secondary), measured by atomic force microscopy at LLNL. The multilayer-coated AIA flight mirrors are well within the peak wavelength tolerance of $335.4 \pm 4.97 \AA$ and peak reflectance goal of $>10 \%$, required in $>80 \%$ of the clear aperture.

The agreement between the model and measured reflectance is excellent for the in-band $\left(1^{\text {st }} \mathrm{Bragg}\right)$ order of all three multilayers shown in Figs. 8, 9 and 10. Some discrepancies exist between models and measured data in the out-of-band wavelength regions of all three samples, especially towards the longer wavelengths. This could be due to imperfections in the multilayer models, especially since the longer wavelengths are reflected by the topmost layers in the multilayer, which include the capping layer and possible oxides and hydrocarbon layers that may have formed on the surface of the aged samples. The exact structure of these top layers is challenging to predict and to model and may thus be partially responsible for the discrepancies. It should be noted that the effect of any oxides in the reflectance of the multilayers is especially pronounced at the longer wavelengths. Another reason for the discrepancies could be the lack of accurate experimental optical constants of materials in the longer wavelength regions. The in-band reflectance of AIA flight witness samples was also measured at beamline 6.3.2. of the ALS shortly after deposition, at the same time as the AIA flight mirrors were measured. A comparison of these measurements with the measurements on aged flight witness samples presented in this Section, will be given in a future publication. 

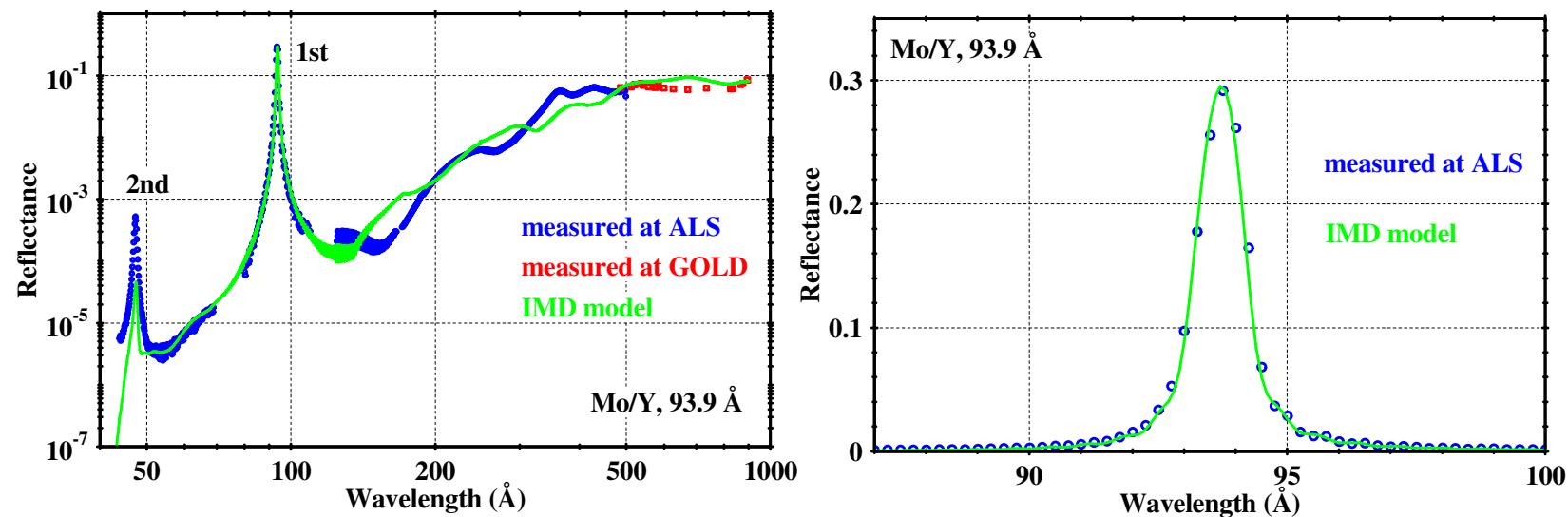

Fig. 8: Measured and modeled reflectance of AIA flight witness multilayer samples, Mo/Y at $93.9 \AA$. To model the measured reflectance, the IMD software ${ }^{14}$ was used with a compilation of experimental optical constants. Left: The in-band and out-of-band reflectance is plotted on logarithmic axes. The $1^{\text {st }}$ and $2^{\text {nd }}$ multilayer Bragg orders are shown. Right: The in-band reflectance $\left(1^{\text {st }}\right.$ Bragg order) is plotted on linear axes.
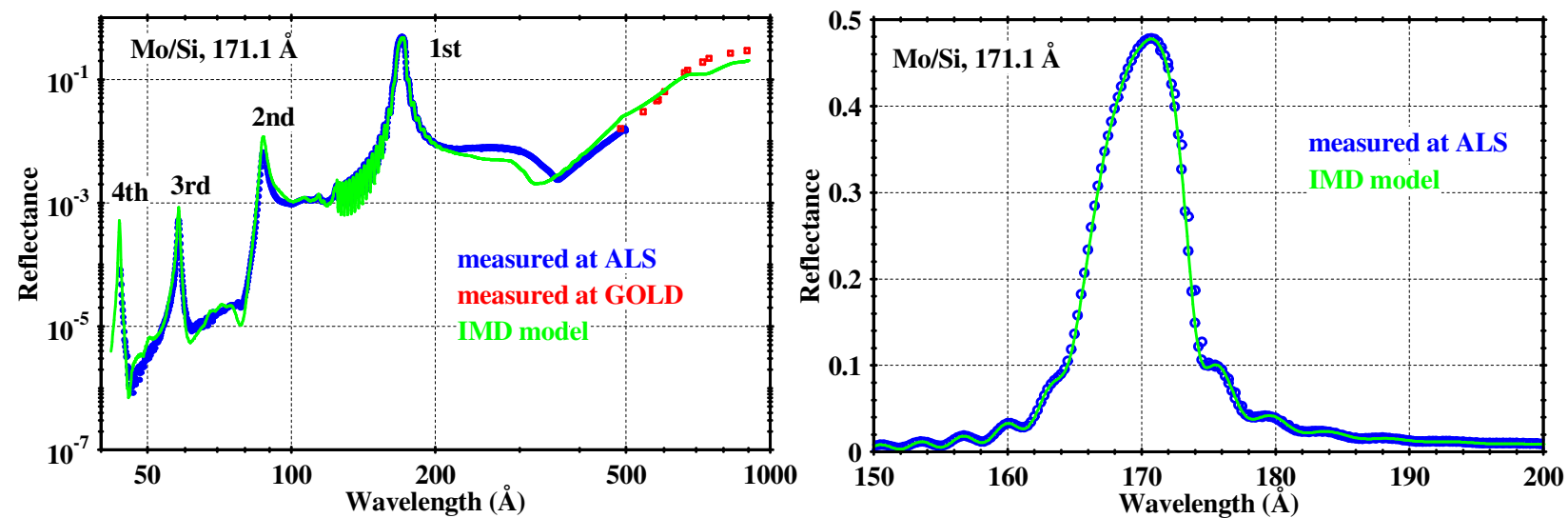

Fig. 9: Measured and modeled reflectance of AIA flight witness multilayer samples, Mo/Si at $171.1 \AA$. To model the measured reflectance, the IMD software ${ }^{14}$ was used with a compilation of experimental optical constants. Left: The in-band and out-of-band reflectance is plotted on logarithmic axes. The $1^{\text {st }}$ through $4^{\text {th }}$ multilayer Bragg orders are shown. Right: The in-band reflectance $\left(1^{\text {st }}\right.$ Bragg order) is plotted on linear axes.
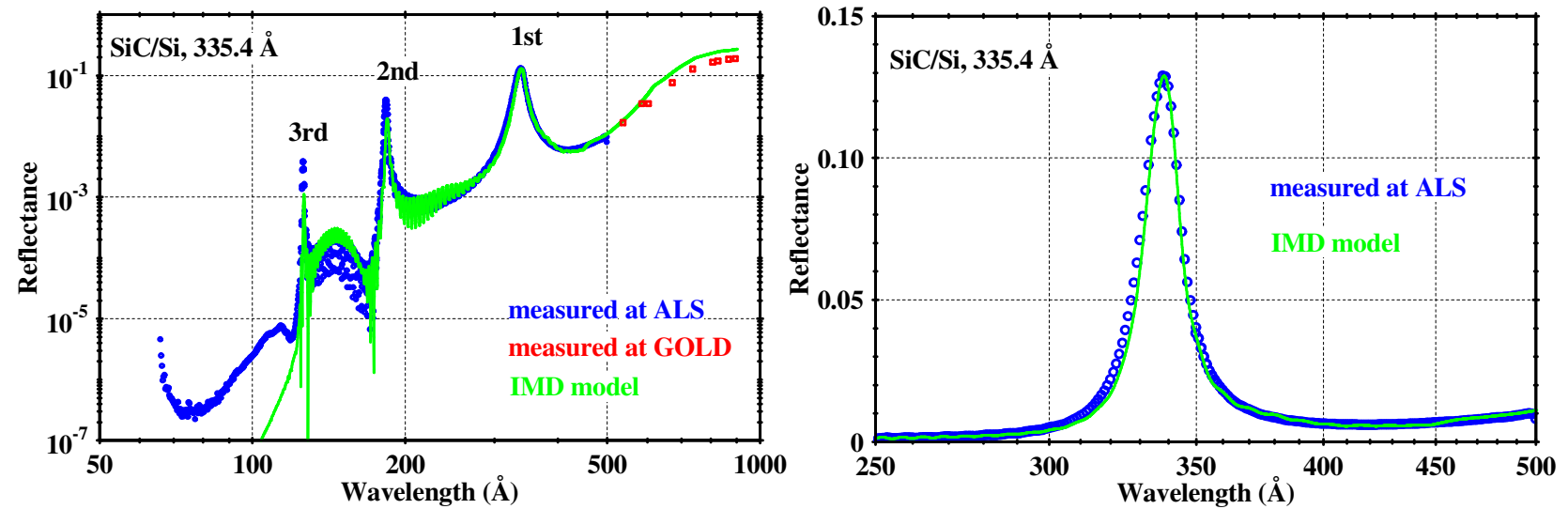

Fig. 10: Measured and modeled reflectance of AIA flight witness multilayer samples, SiC/Si at $335.4 \AA$. To model the measured reflectance, the IMD software ${ }^{14}$ was used with a compilation of experimental optical constants. Left: The in-band and out-of-band reflectance is plotted on logarithmic axes. The $1^{\text {st }}$ through $3^{\text {rd }}$ multilayer Bragg orders are shown. Right: The in-band reflectance $\left(1^{\text {st }}\right.$ Bragg order) is plotted on linear axes. 


\section{SUMMARY}

The in-band and out-of-band calibration of flight mirrors and witnesses for three wavelength channels of the AIA instrument aboard SDO is presented in this manuscript. It is shown that the AIA multilayer mirrors meet all specifications. The in-band results of the AIA flight mirror calibrations have been included in the initial photometric calibration of the AIA instrument. The in-band and out-of-band measurements in the 40-900 ̊ wavelength region, obtained on aged AIA flight witness samples, will be included in future photometric calibrations of the AIA instrument. This is the first time that in-band and out-of-band EUV reflectance measurements and models for $\mathrm{Mo} / \mathrm{Y}, \mathrm{Mo} / \mathrm{Si}$ and $\mathrm{SiC} / \mathrm{Si}$ multilayers are reported in such an extended wavelength range. These results are expected to further improve the understanding of the AIA instrument response and the corresponding interpretation of data from the AIA instrument.

\section{ACKNOWLEDGEMENTS}

This work was performed under the auspices of the U.S. Department of Energy by Lawrence Livermore National Laboratory under Contract DE-AC52-07NA27344 and by the University of California Lawrence Berkeley National Laboratory under Contract No. DE-AC03-76F00098. Funding for a portion of this work was provided by the Smithsonian Astrophysical Observatory. The Advanced Light Source is supported by the Director, Office of Science, Office of Basic Energy Sciences, of the U.S. Department of Energy under Contract No. DE-AC02-05CH11231. GOLD acknowledges financial support from the National Programme for Space Research, Subdirección General de Proyectos de Investigación, Ministerio de Ciencia y Tecnología, project number AYA2010-22032.

\section{REFERENCES}

${ }^{1}$ J. R. Lemen, et al, Solar Physics, 275, 41-66, (2012).

${ }^{2}$ P. Boerner, et al, Solar Physics 275, 17-40, (2012).

${ }^{3}$ For more information, see the official SDO site at http://sdo.gsfc.nasa.gov/ and AIA sites at http://hea-www.harvard.edu/AIA/ and http://aia.lmsal.com/

${ }^{4}$ R. Soufli, D. L. Windt, J. C. Robinson, E. A. Spiller, F. J. Dollar, A. L. Aquila, E. M. Gullikson, B. Kjornrattanawanich, J. F. Seely, L. Golub, Proc. SPIE 5901, 59010M (2005).

${ }^{5}$ R. Soufli, M. Fernández-Perea, J. C. Robinson, S. L. Baker, "Corrosion-resistant multilayer coatings for the 25-75 nm wavelength region", 2011 International Workshop on EUV and Soft X-ray Sources, University College Dublin, Ireland, November 7-9, 2011. http://www.euvlitho.com/2011/S17.pdf

${ }^{6}$ R. Soufli, M. Fernández-Perea, S. L. Baker, J. C. Robinson, J. Alameda, C. C. Walton, Appl. Phys Lett. 101043111 (2012).

${ }^{7}$ M. Fernández-Perea, R. Soufli, J. C. Robinson, L. Rodríguez-de Marcos, J. A. Mendez, J. I. Larruquert and E. M. Gullikson, submitted to Optics Express (2012).

${ }^{8}$ R. Soufli, M. Fernández-Perea, J. C. Robinson, S. L. Baker, J. Alameda, E. M. Gullikson, “Corrosion-resistant, highperformance $\mathrm{Mg} / \mathrm{SiC}$ multilayer coatings for solar physics in the $25-80 \mathrm{~nm}$ wavelength region", Proc. SPIE 8443 (2012), this Conference Proceedings.

${ }^{9}$ R. Soufli, R. M. Hudyma, E. Spiller, E. M. Gullikson, M. A. Schmidt, J. C. Robinson, S. L. Baker, C. C. Walton, and J.S. Taylor, Appl. Opt. 46, 3736 (2007).

${ }^{10}$ D. L. Windt and W. K. Waskiewicz, J. Vac. Sci. Technol. B 12, 3826-3832 (1994).

${ }^{11}$ J. H. Underwood, E. M. Gullikson, J.Electr. Spectr. Rel. Phenom. 92, 265-272 (1998).

${ }^{12}$ E. M. Gullikson, S. Mrowka, B. B. Kaufmann, Proc. SPIE 4343, 363-373 (2001).

${ }^{13}$ R. Soufli, S. L. Baker, D. L. Windt, J. C. Robinson, E. M. Gullikson, W. A. Podgorski, L. Golub, Appl. Opt. 46, 31563163 (2007).

${ }^{14}$ D. L. Windt, Computers in Physics 12, 360 (1998).

${ }^{15}$ B. L. Henke, E. M. Gullikson, and J. C. Davis, Atomic Data and Nuclear Data Tables 54, 181-342 (1993).

${ }^{16}$ R. Soufli and E. M. Gullikson, Appl. Opt. 37, 1713-1719 (1998).

${ }^{17}$ C. Tarrio, R. N. Watts, T. B. Lucatorto, J. M. Slaughter, and C. M. Falco, Appl. Opt. 37, 4100-4104 (1998).

${ }^{18}$ J. I. Larruquert and R. A. M. Keski-Kuha, Appl. Opt. 39, 2772-2781 (2000). 
${ }^{19}$ D. L. Windt, W. C. Cash, Jr., M. Scott, P. Arendt, B. Newnam, R. F. Fisher, and A. B. Swartzlander, Appl. Opt. 27, 246-278 (1988).

${ }^{20}$ B. Sae-Lao and R. Soufli, Appl. Opt. 41, $7309-7316$ (2002).

${ }^{21}$ J. H. Weaver and C. G. Olson, Phys. Rev. B 15 590-594 (1977).

${ }^{22}$ E. M. Gullikson, P. Denham, S. Mrowka, and J. H. Underwood, Phys. Rev. B 49, 16283-16288 (1994).

${ }^{23}$ R. Soufli and E. M. Gullikson, Appl. Opt. 36, 5499-5507 (1997).

${ }^{24}$ W. R. Hunter (unpublished), included in the Handbook of Optical Constants of Solids, E. D. Palik editor (Academic Press, 1997).

${ }^{25}$ H. R. Philipp, J. of Physics and Chemistry of Solids 32, 1935-1945 (1971).

${ }^{26}$ E. Filatova, V. Lukyanov, R. Barchewitz, J-M André, M. Idir and Ph. Stemmler, J. Phys.: Condens. Matter 11 33553370 (1999).

${ }^{27}$ W. R. Hunter (unpublished), included in the Handbook of Optical Constants of Solids, E. D. Palik editor (Academic Press, 1997).

${ }^{28}$ C. Tarrio and S. E. Schnatterly, JOSA B 10, 952-957 (1993).

${ }^{29}$ J. I. Larruquert, A. P. Pérez-Marín, S. García-Cortés, L. Rodríguez-de Marcos, J. A. Aznárez, and J. A. Méndez, JOSA A 28, 2340-2345 (2011).

${ }^{30}$ D. L. Windt, W. C. Cash, Jr., M. Scott, P. Arendt, B. Newnam, R. F. Fisher, A. B. Swartzlander, P. Z. Takacs, and J. M. Pinneo, Appl. Opt. 27, 279-295 (1988). 